\title{
Magnetic poles and dipole tilt variation over the past decades to millennia
}

\author{
M. Korte and M. Mandea \\ GeoForschungsZentrum Potsdam, Telegrafenberg, 14473 Potsdam, Germany \\ (Received December 6, 2007; Revised July 2, 2008; Accepted August 2, 2008; Online published October 15, 2008)
}

\begin{abstract}
Due to the strong dipolar character of the geomagnetic field the shielding effect against cosmic and solar ray particles is weakest in the polar regions. We here present a comprehensive study of the evolution of magnetic and geomagnetic pole locations and the Earth's magnetic core field in both polar regions over the past few years to millennia. North and south magnetic poles change independently according to the asymmetric complexity of the field in the two hemispheres, and the changes are not correlated to variations of the dipole axis. The recent, strong acceleration of the north magnetic pole motion appears to be linked to a reverse flux patch at the coremantle boundary, and an increasing deceleration of the pole over the next years seems likely. Over the whole studied period of 7000 years two periods of comparatively high velocity of the north magnetic pole are observed at $4500 \mathrm{BC}$ and $1300 \mathrm{BC}$, based on the presently available data and models. Geographic latitudes and longitudes of magnetic and geomagnetic poles based on the studied geomagnetic field models are available together with animations of the poles and polar field behaviour from our webpage http://www.gfz-potsdam.de/geomagneticfield/poles.
\end{abstract}

Key words: Geomagnetic field, magnetic poles, dipole axis.

\section{Introduction}

The geomagnetic main field, generated in the Earth's outer fluid core, is constantly changing, this temporal variation being known as secular variation. At and above the Earth's surface the observed time scales of secular variation range from about one year (geomagnetic jerks) to millions of years, where extreme events like excursions and polarity reversals occur. The field is approximately dipole-shaped in the near-Earth environment. Recently, a strong decrease of the dipole moment, persisting at least since the beginning of systematic, full vector field measurements around 1830, has raised attention (Gubbins, 1987; Hulot et al., 2002; Olson, 2002; Constable and Korte, 2006; Gubbins et al., 2006). Another feature of the field is currently showing quite extreme changes, and this is the magnetic north pole location (Newitt et al., 2002). Since about 1970 its velocity has suddenly increased to almost $60 \mathrm{~km} / \mathrm{yr}$, much higher than the average observed over the past centuries (Mandea and Dormy, 2003).

The polar regions are the areas where incoming cosmic and solar wind particles, being deflected by and following the geomagnetic field lines, penetrate most deeply into the atmosphere. Connections between geomagnetic field and climate changes have once again become discussed controversially lately (Courtillot et al., 2007; Bard and Delaygue, 2007). If mechanisms like galactic cosmic rays influencing cloud cover play a role, then the location of the geomagnetic field poles, particularly with regard to latitude and/or relative to land mass, might be important in terms of cli-

Copyright (c) The Society of Geomagnetism and Earth, Planetary and Space Sciences (SGEPSS); The Seismological Society of Japan; The Volcanological Society of Japan; The Geodetic Society of Japan; The Japanese Society for Planetary Sciences; TERRAPUB. matological effects. The behaviour of the magnetic poles is investigated in the present study together with the general main field behaviour in the polar regions. Part of our motivation also comes from the recently increased interest in the Arctic and Antarctic during the International Polar Year (IPY) which started in March 2007.

Two sets of poles are defined with respect to the magnetic field, the magnetic and the geomagnetic poles, with characteristics summarised in the following. The magnetic pole or dip pole positions are the areas where the field lines penetrate the Earth vertically, i.e. the inclination reaches $90^{\circ}$. Due to the non-dipole influences in the core field, north (NMP) and south (SMP) magnetic poles are not diametrically opposed. Their locations can be determined in two ways, by direct measurements or from global geomagnetic field models. Direct observations involve costly expeditions to inhospitable areas, as currently the NMP is situated far away on the icy Arctic Ocean, and the SMP is in the Ocean, just off the Antarctic coast and south of Australia (see Fig. 1). Measurements can be influenced by local crustal field anomalies. Moreover, due to the strong influence of external magnetic field contributions at high geomagnetic latitudes the magnetic pole locations are not fixed points, but move within a radius of a couple of $\mathrm{km}$ up to $100 \mathrm{~km}$ per day, depending on geomagnetic activity (Dawson and Newitt, 1982). For this reason, measurements to determine the magnetic poles of the core field have to be properly designed to account not only for the specific conditions in the Arctic and Antarctic regions, but also for the high variability of the vertical field lines locations. Eight direct measurements of the pole locations for the northern hemisphere have been carried out $(1831,1905,1947,1962$, 1973, 1984, 1994 and 2001, see Newitt and Barton, 1996; 


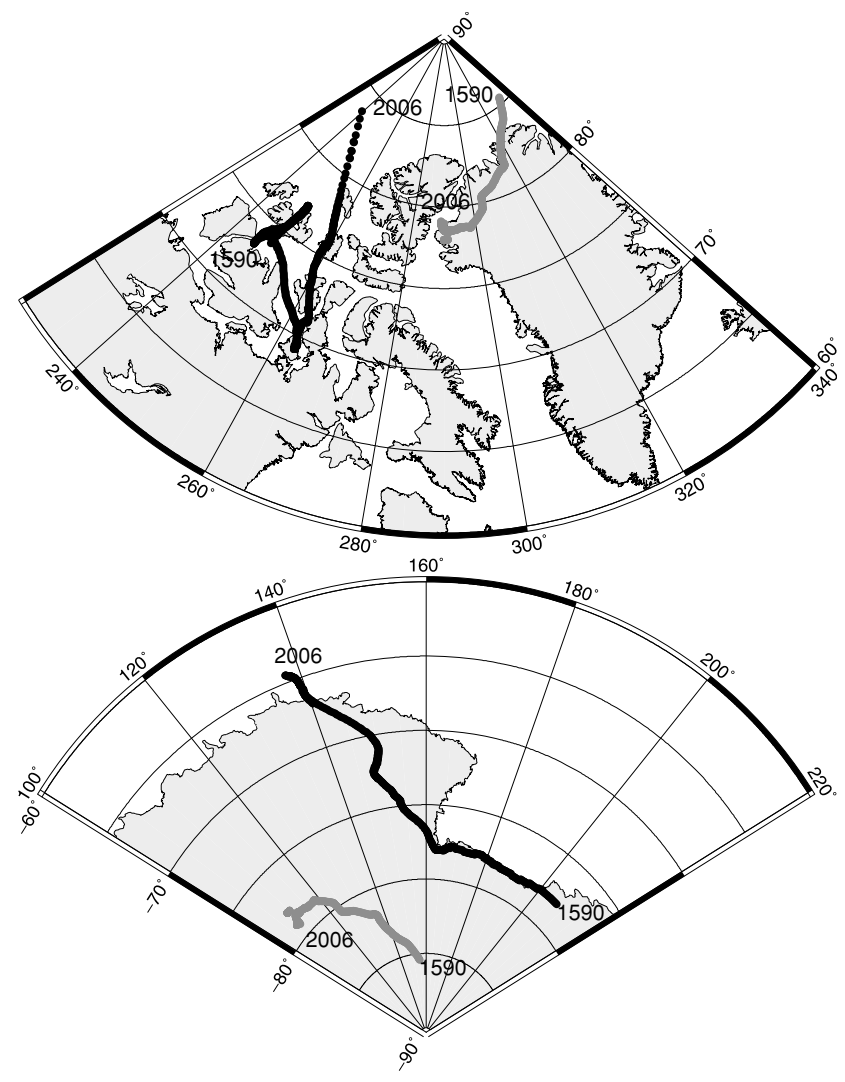

Fig. 1. Location of NMP (left) and SMP (right) from gufm1 (1590-1990), CM4 (1960-2002) and CHAOS (2000-2006) in black, and geomagnetic poles from gufm1, CM4 and CHAOS in gray.

Newitt et al., 2002; Mandea and Dormy, 2003) and five for the southern hemisphere (1909, 1912, 1952, 1986 and 2000, see Barton, 1987, 1988, 2002; Mandea and Dormy, 2003). Of course, it is easier to determine the magnetic pole locations from global spherical harmonic geomagnetic field models. However, in this case the accuracy depends on the model quality, which largely depends on the availability of a good global data coverage, data accuracy, and also separation between the internal and external field contributions. The predictions from modern global field models agree well with measured magnetic pole positions (e.g. Mandea and Dormy, 2003).

The geomagnetic poles are the poles of the approximated dipole field, i.e. the intersections between the dipole axis and the Earth's surface. In contrast to the magnetic poles, geomagnetic north (NGP) and south (SGP) pole are diametrically opposed and move simultaneously. They can conveniently be determined from global spherical harmonic field models, but they cannot be directly measured from any measurements at the Earth's surface or near-Earth satellite altitudes in the polar regions only.

Note a potential cause of confusion regarding (geo-) magnetic poles: according to the physical definition, with respect to the direction of magnetic field lines, the poles on the northern hemisphere are south poles and vice versa. This is confusing in a geographic reference frame, and in the following we ignore the physical definition and use the terms "north pole" for those in the northern and "south pole" for those in the southern hemisphere.
Changes of the magnetic poles must be closely linked to changes of the magnetic field in the polar regions. Geodynamo theory predicts different fluid flow behaviour inside and outside the tangent cylinder (TC), an imaginary cylinder tangent to the inner core equator and parallel to the Earth's rotation axis (see e.g. Carrigan and Busse, 1983; Cardin and Olson, 1994; Aurnou et al., 2003; Dormy et al., 2004). The fluid motion in the outer core generally should be nearly two-dimensional (2D), i.e. quasigeostrophic, according to the Taylor-Proudman theorem. Theory predicts that both small-scale and large-scale convective motion could exist within the TC (Gubbins and Bloxham, 1987). Secular variation studies using data from the past 20 to 150 yr (Olson and Aurmou, 1999; Pais and Hulot, 2000; Hulot et al., 2002) propose polar vortices as evidence for effects of the TC.

In the following, we first investigate changes of the magnetic and geomagnetic poles and then study the behaviour of the overall field distribution in the polar regions, all based on spherical harmonic geomagnetic field models. We successively go back in time, from the high-resolution but short time picture to the millennial scale development available only with low spatial and temporal resolution.

\section{Methods and Models}

Temporally continuous geomagnetic field models are ideally suited to study the behaviour of magnetic and geomagnetic poles over the past years to millennia. All models we use are based on spherical harmonic expansions in space with the continuous temporal representation provided by expanding each Gauss coefficient in a cubic B-splines representation in time. Assuming no sources in the region of observation, the time-dependent geomagnetic main field $\mathbf{B}(t)$ is described as the gradient of a scalar potential $V(t)$,

$$
\mathbf{B}(t)=-\nabla V(t) .
$$

This can be expanded into a series of spherical harmonic functions,

$$
\begin{aligned}
V(r, \theta, \phi, t)= & a \sum_{l=1}^{L} \sum_{m=0}^{l} \sum_{k=1}^{K}\left(\frac{a}{r}\right)^{l+1} \\
& \cdot\left[g_{l}^{m, k} \cos (m \phi)+h_{l}^{m, k} \sin (m \phi)\right] \\
& \cdot P_{l}^{m}(\cos \theta) M_{k}(t)
\end{aligned}
$$

where $(r, \theta, \phi)$ are spherical polar coordinates and $a=$ $6371.2 \mathrm{~km}$ is the mean radius of the Earth's surface. The $P_{l}^{m}(\cos \theta)$ are the Schmidt quasi-normalised associated Legendre functions of degree $l$ and order $m$. The coefficients $\left\{g_{l}^{m, k}, h_{l}^{m, k}\right\}$ are related to the standard Gauss coefficients $\left\{g_{l}^{m}, h_{l}^{m}\right\}$ for a single epoch $t$ by

$$
g_{l}^{m}(t)=\sum_{k=1}^{K} g_{l}^{m, k} M_{k}(t)
$$

by the splines $M_{k}(t)$, and the same for $h_{l}^{m}$. The spherical harmonic expansion allows the study of the magnetic field distribution and change not only in the regions of measurements at and above the Earth's surface, but also at the coremantle boundary (CMB) assuming an insulating mantle by 
simple downward continuation via the relation of the radii $\left(\left(\frac{a}{r}\right)^{l+1}\right)$.

We consider four continuous field models in this study to investigate pole and field changes over different time-scales.

- CHAOS is the first continuous model based on satellite data only, covering the time interval from March 1999 to December 2005 (Olsen et al., 2006). The name represents the three satellites which have provided data: CHAMP, Ørsted, SAC-C. The CHAOS model describes the core field and its secular variation with the highest possible spatial and temporal resolution. The core field is fully resolved up to degrees where its contribution is hidden by the crustal field. Secular variation is reliably resolved up to spherical harmonic degree and order 15, unprecedented by any other continuous field model. The knot-point spacing of the splines is $1 \mathrm{yr}$.

- CM4, the Comprehensive Model (4th generation), is an attempt to describe all field contributions in one model, from core, lithosphere, sources external to the Earth and their induced counterparts (Sabaka et al., 2004). It covers the time span 1960 to mid-2002 with a spline knot-point spacing of $2.5 \mathrm{yrs}$. This model can be useful for separating certain contributions of the quiet time magnetic field. Here, we only consider the core field and its secular variation given by this model.

- Gufm1 goes as far back in time as direct magnetic field measurements allow, covering the interval 1590 to 1990 (Jackson et al., 2000) with spline knot-points every $2.5 \mathrm{yrs}$. This is the latest achievement of the pioneering work by Bloxham and Jackson (1992) to develop spline-based continuous models, which also are particularly useful for studying the geomagnetic field at the CMB. In order not to include artificial structure caused by data errors and insufficient data coverage, a regularisation has been applied in the modelling. The result is a smoother model which shows the minimum amount of structure necessary to explain the used data. As a consequence, the spatial resolution of this model clearly changes and improves with time due to increasing amounts of (better) data. Comparing geomagnetic power spectra we estimate the reliable spatial resolution of main field and secular variation to increase from about degree 6 and 5, respectively, in 1590 to 10 (main field) and 7 (secular variation) for the last years of the model.

- CALS7K.2, a Continuous model based on Archaeomagnetic and Lake Sediment data of the past $7 \mathrm{kyr}$, is one of the first attempts to extend continuous global field models towards paleomagnetic time-scales with resolution beyond the quadrupole contribution (Korte and Constable, 2005a). Although the modelling technique is nearly the same as for gufm 1 , the resolution is severely limited by the inferior data distribution and uncertainties in the archaeo- and paleomagnetic data and their dating, which are much higher than even in historical direct field observations. A similar regularisation as in gufm 1 has been applied and the reliable spatial resolution of this model is about spherical har- monic degree 4 to 5 , while the temporal resolution is no better than 50 to $100 \mathrm{yr}$ (spline knot-points are separated by about $50 \mathrm{yrs}$ ).

Geomagnetic north pole longitude $\phi$ and latitude $\lambda$ are conveniently found from spherical harmonic dipole coefficients $g_{1}^{0}, g_{1}^{1}$ and $h_{1}^{1}$ following Langel (1987)

$$
\tan \phi=\frac{h_{1}^{1}}{g_{1}^{1}}
$$

and

$$
\begin{aligned}
\lambda & =90^{\circ}-\theta, \\
\cos \theta & =\frac{g_{1}^{0}}{m},
\end{aligned}
$$

with

$$
m=\sqrt{\left(g_{1}^{0}\right)^{2}+\left(g_{1}^{1}\right)^{2}+\left(h_{1}^{1}\right)^{2}} .
$$

Geomagnetic south pole locations are obtained straightforwardly as the diametrically opposed points. Magnetic poles predicted by the models are found by evaluating the full core field description in the north and south polar regions and finding the locations where inclination values are $90^{\circ}$. The velocity, $v(t)$ of the pole movement at time $t=\left(t_{1}+t_{2}\right) / 2$ is calculated as

$v(t)=\frac{\left(\left[\left(\lambda\left(t_{2}\right)-\lambda\left(t_{1}\right)\right) \frac{2 \pi R_{\mathrm{pol}}}{360^{\circ}}\right]^{2}+\left[\left(\phi\left(t_{2}\right)-\phi\left(t_{1}\right)\right) \frac{2 \pi R_{\mathrm{eq}}}{360^{\circ}} \cos \left(\lambda\left(t_{2}\right)\right)\right]^{2}\right)^{\frac{1}{2}}}{t_{2}-t_{1}}$

with $R_{\text {pol }}=6356.8 \mathrm{~km}$ and $R_{\text {eq }}=6378.2 \mathrm{~km}$ the polar and equatorial radius of the Earth, respectively.

Computer-readable ASCII files with geographic latitudes and longidutes of all the magnetic and geomagnetic pole locations discussed in this work are provided on our webpage http://www.gfz-potsdam.de/geomagnetic-field/poles.

\section{Migration of the Magnetic and Geomagnetic Poles}

In the following we first describe the behaviour of the geomagnetic (i.e. dipole axis) poles, and then of the magnetic poles, for the last four centuries coverd by models based on direct field observations (Section 3.1). The same is done for the past 7 millennia, when only indirect data can be used for modelling, in Section 3.2.

\subsection{From decades to centuries}

The changes of magnetic and geomagnetic pole positions since 1590 are shown in Fig. 1. The corresponding velocities together with latitudinal and longitudinal changes are given in Fig. 2. The dipole axis has been tilting away from the geographical axis for most of the time, while turning slightly westward in the northern hemisphere. Between 1800 and 1950 its latitude remained nearly constant and for the past five decades the axis tilt has been slowly decreasing. The velocity of this movement has generally slightly decreased between 1590 and 1900 and increased afterwards, possibly somewhat correlated to increasing and 

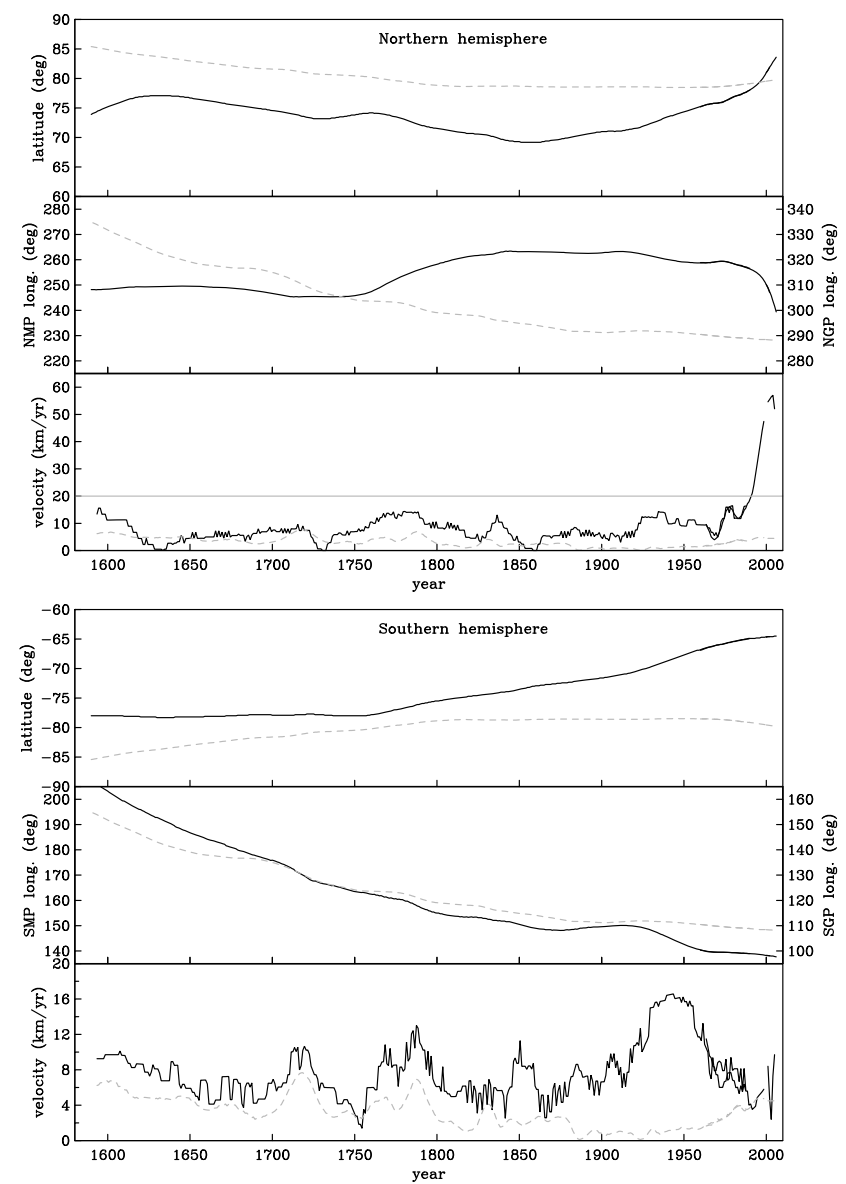

Fig. 2. Latitude, longitude and velocity of NMP (top) and SMP (bottom). Solid black lines are the magnetic poles, dashed gray lines the geomagnetic ones, from models gufm1 (1590-1990), CM4 (1960-2002) and CHAOS (2000-2006). The pole velocities computed from the gufm1, CM4 and CHAOS models are smoothed by running averages of five (gufm1) and two (CM4, CHAOS) years, respectively. Note the different ordinate scales for the velocities, where the gray line indicates the top of the southern hemipshere axis.

decreasing axis tilt. The velocity ranges between nearly 0 and $8 \mathrm{~km} / \mathrm{yr}$ with two maxima around 1718 and 1787.

The magnetic poles change more quickly and show a strikingly different behaviour on northern and southern hemisphere. The SMP has been drifting west since 1590 with only slight changes in direction. These occurred in 1750 , when the magnetic pole started to move away from the geographic pole and twice in the early 20th century. The velocity generally varies between 2 and $12 \mathrm{~km} / \mathrm{yr}$ with a maximum of about $14 \mathrm{~km} / \mathrm{yr}$ lasting for 4 decades around 1950. Interestingly, the velocities of south magnetic and geomagnetic poles change quite simultaneously up to 1820 , but not afterwards.

The NMP shows the most vivid behaviour with several pronounced changes of direction. The longitude displays a step-like behaviour, remaining nearly constant over the first time interval, until 1750. Thereafter, in less than $50 \mathrm{yr}$ the longitude is shifted by nearly $20^{\circ}$ east. After another stable period, towards the end of 20th century an even more sudden longitudinal change to the west is observed. The latitude increases until 1630 and then decreases until 1860 with a slight interruption from 1730 to 1760 . Since 1860 the
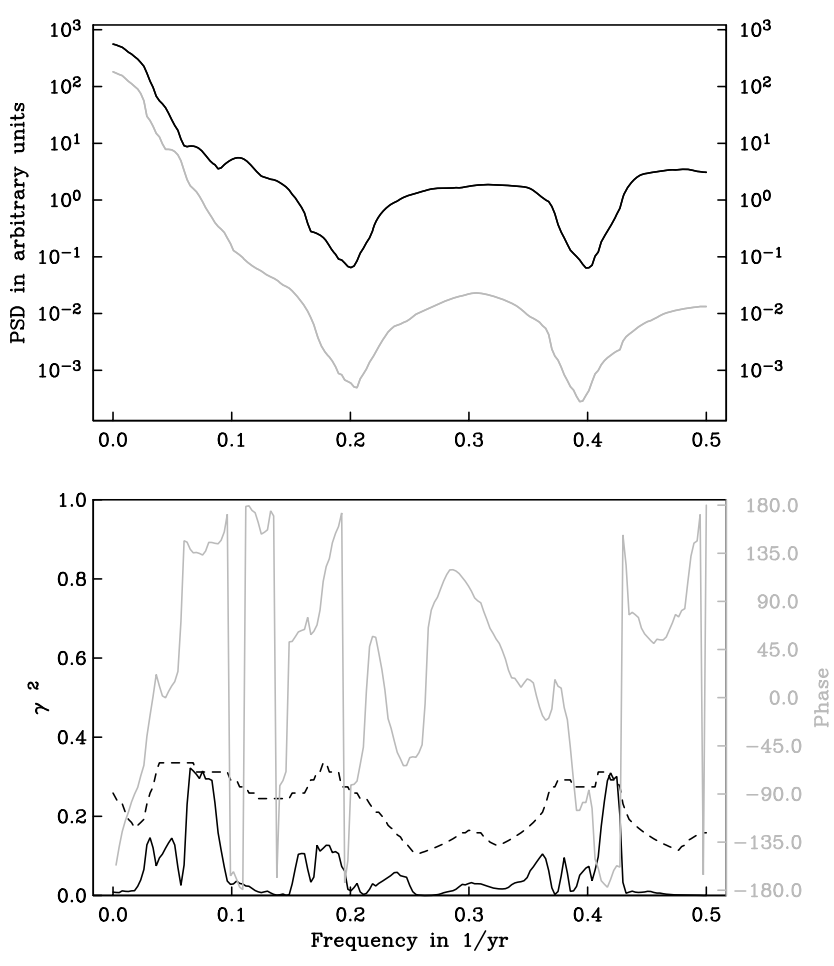

Fig. 3. Power spectral densities (PSD) of the NMP (black) and NGP (gray) velocity of gufm1 (top) and their cross correlation (bottom, black line) and phase shift (bottom, gray line). The dashed black line gives the 95\% confidence level below which the two series are uncorrelated.

latitude of the magnetic pole increases. An acceleration of this northward movement together with the onset of a strong westward component to it has led to a remarkable change of velocity. This has increased dramatically since 1970 , after nearly $400 \mathrm{yr}$ of small variations, around some $5 \mathrm{~km} / \mathrm{yr}$, within a similar range as that of the SMP. Currently the NMP moves north-westward at a speed of about $60 \mathrm{~km} / \mathrm{yr}$. The CHAOS model suggests, however, that the velocity has recently started to decrease. This is more clearly shown in a recent study by Olsen and Mandea (2007) from an improved version of the CHAOS model.

We performed analyses of power spectral densities, using the adaptive multi-taper method of Riedel and Sidorenko (1995) and program "cross" by R. L. Parker. None of the velocities shows any clear periodicities. We also found no significant cross correlations, neither between any of the magnetic and the geomagnetic pole velocities, nor between NMP and SMP velocities. As an example, Fig. 3 shows power spectral densities of the the NMP and NGP velocities from the model gufm1 and their cross-correlation. The coherence is below the significance level for all periods. The power spectral densities show a lack of power at periods of 5 and 2.5 years due to the smoothing filter used in calculating the velocities, but no signifcant periodicities otherwise.

\subsection{Over the past 7 millennia}

Figures 4 and 5 show location and velocity of the poles over the past $7 \mathrm{kyr}$ based on the CALS7K.2 model and compared to gufm 1 for the overlapping time. The geomagnetic poles, being a property of the dipole field, should be resolved well in CALS7K. This is confirmed by the good agreement between the two models between 1590 and 1750 . 

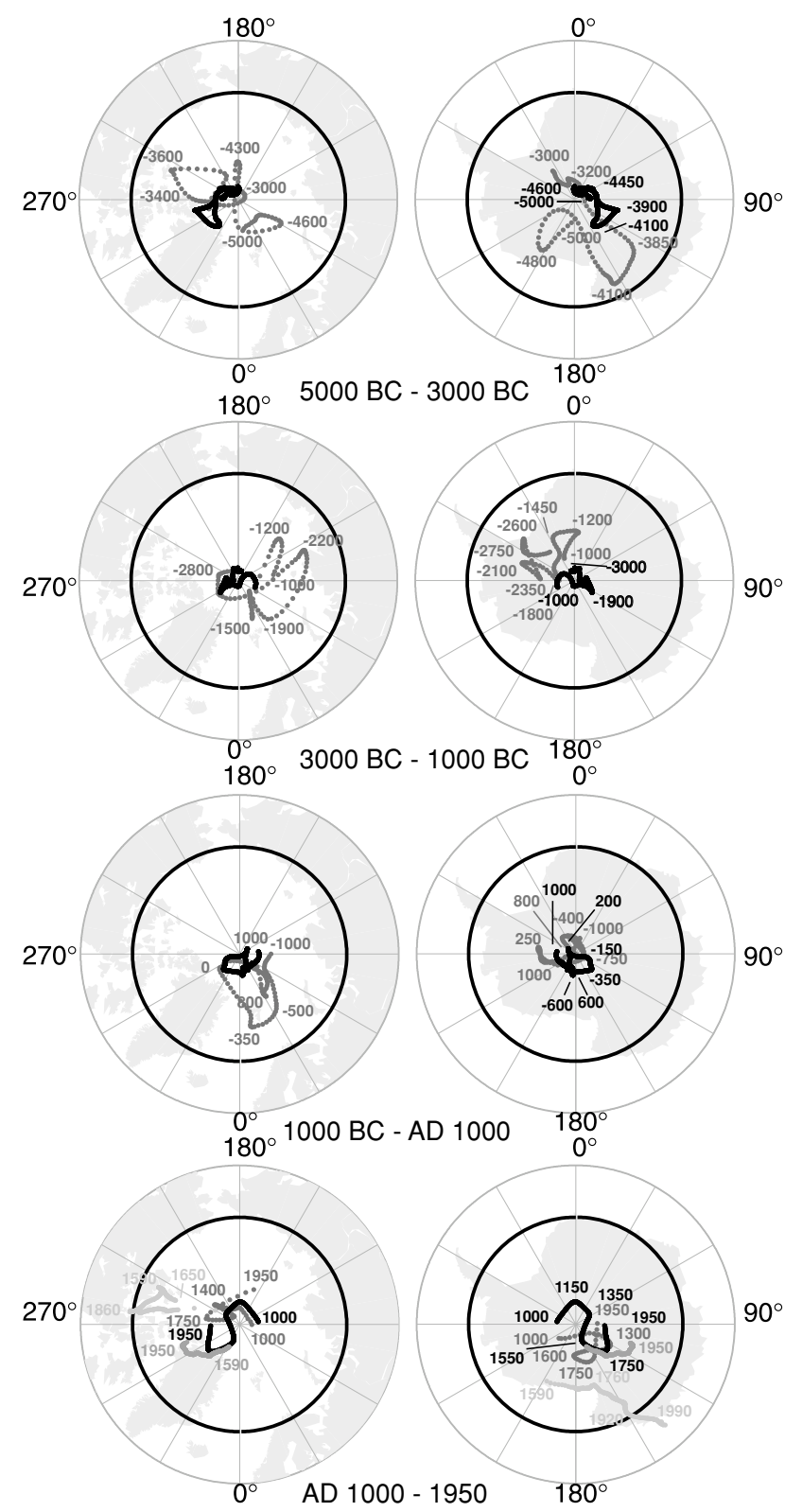

Fig. 4. Location of poles from $5000 \mathrm{BC}$ to 1990 for north (left) and south (right) hemisphere (polar caps shown to $60^{\circ} \mathrm{N}$ and $\mathrm{S}$, respectively). Gray and black are magnetic and geomagnetic pole positions, respectively, as given by CALS7K.2. Medium and light gray in the bottom panels are, again, magnetic and geomagnetic pole positions from gufm 1 from 1590 to 1990 . The numbers mark approximate years of significant directional changes. For the simultaneous movements of the geomagnetic poles these labels are only given in the southern hemisphere panels. The thick black latitudinal circle is the boundary of the inner core TC, projected to the Earth's surface from the CMB.

The deviation when compared to gufm 1 , in the most recent $150 \mathrm{yr}$, is due to temporal edge effects in the continuous model (see Korte and Constable, 2008). A similar effect has to be expected in the earliest centuries covered by the CALS7K.2 model. The dipole axis remained rather close to the rotation axis over most of the time interval, never tilting by more than $10^{\circ}$ until 1800 and tilting by more than $5^{\circ}$ only twice, at about $4000 \mathrm{BC}$ and from 1600 on. The tilt occurs in all directions over time, with both westward and eastward movements of the axis. In summary, eastward movements dominate over the whole $7 \mathrm{kyr}$. The velocity of dipole axis
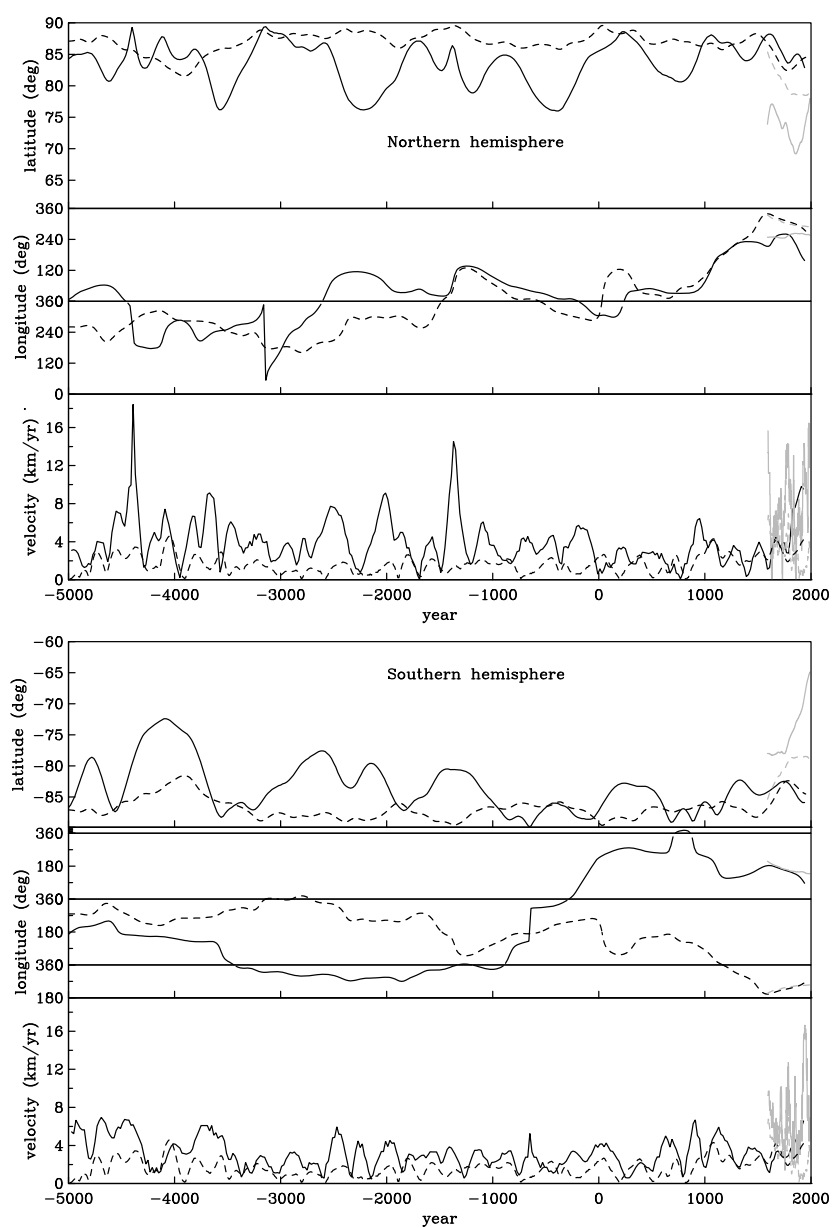

Fig. 5. Latitude, longitude and velocity of the north (top) and south (bottom) poles since $5000 \mathrm{BC}$ from model CALS7K.2. Solid lines are magnetic poles, dashed lines the geomagnetic ones, and gray, respectively, the same again from gufm1. Abrupt changes of magnetic poles longitude about $3150 \mathrm{BC}$ (north) and $650 \mathrm{BC}$ (south) occur when the poles cross the geographic poles.

motion varies irregularly about low values up to $5 \mathrm{~km} / \mathrm{yr}$, similar to the behaviour during the recent centuries.

The magnetic pole locations obtained from the $7 \mathrm{kyr}$ model have to be regarded with caution because they are influenced by smaller scale field contributions and might not be resolved well over the millennial scale. This becomes obvious from the clear disagreement between the CALS7K. 2 and gufm1 pole predictions. Nevertheless, we briefly report the model predictions here, but discuss their relevance in Section 5. According to the CALS7K.2 model both NMP and SMP never moved to latitudes lower than $73^{\circ} \mathrm{N}$ and $\mathrm{S}$, respectively, during the past millennia. All longitudes are involved and even in the strongest deviations from the geographical pole no preference for certain longitudinal regions can be detected. Rather abrupt longitudinal changes can occur naturally when the pole latitude is close to $90^{\circ}$, as for example around $3150 \mathrm{BC}$ or $650 \mathrm{BC}$ in the northern or southern hemisphere, respectively. The velocities of NMP and SMP given by CALS7K.2 are low all the time, with values no higher than $7 \mathrm{~km} / \mathrm{yr}$ for the SMP and in general less than $10 \mathrm{~km} / \mathrm{yr}$ for the NMP, with only two exceptions around $4500 \mathrm{BC}$ and $1300 \mathrm{BC}$, where maximum velocities between 15 and $20 \mathrm{~km} / \mathrm{yr}$ are estimated. 


\section{Polar Field Evolution}

The movements of the magnetic poles originate from changes in the polar field morphology, so in this section we study the changes of the radial field component with time. To get a detailed picture of the polar fields of purely internal origin is a difficult task. Firstly, there are no long series of ground measurement data from these regions and young paleo-/archeomagnetic data from there are also sparse. Therefore, the resolution of long-term models might be particularly limited in these areas. Secondly, despite the data selection criteria the recent high-resolution field models based on satellite data are not completely free of external contributions, particularly in the high-latitude regions where these influences are strongest. Small-scale details therefore might be somewhat distorted from external field residuals in the data. These limitations should be kept in mind when we now investigate the behaviour of the core field and its secular variation, based again on the four models, both at the Earth's surface and the CMB. For this, we computed the core field of the CHAOS and CM4 models to maximum spherical harmonic degree 12 , to avoid influences from the crustal field. For gufm 1 and CALS7K. 2 similar limitations are not necessary, because the regularisation applied in these models depletes the influence of spherical harmonic degrees beyond the effective resolution. Figure 6 in this section combines information about the CMB and the Earth's surface. The position of the magnetic poles is mainly of interest at the Earth's surface, while we investigate the field morphology generating these poles at the CMB. The panels show the radial field at the CMB with the TC indicated by a circle. For reference, the continent outlines are overlain, and these and the TC circle represent the area at the Earth's surface which is influenced by the respective areas of the core field if we assume no significant influences across the borders of a cone from the center of the Earth. This allows us to include the position of the magnetic poles at the Earth's surface in the same panels.

At the Earth's surface and in the northern polar region the radial component of the magnetic field is currently characterised by two maxima, situated in the Siberian and Canadian region. They correspond to two prominent areas of strong magnetic flux at the CMB (Bloxham and Gubbins, 1985), more or less just outside the TC (Gubbins and Bloxham, 1987). Two patches of reversed magnetic flux with respect to the dominating dipole direction are identified within the TC at the CMB (Fig. 6(a)).

The southern hemisphere field morphology is significantly different (Mandea and Dormy, 2003). At the Earth's surface, one field maximum centred between Australia and Antarctica, i.e. the expected appearance of a tilted dipole field, is observed. This is accompanied by the large area of anomalously weak field, known as the South Atlantic Anomaly, which reaches well into the near-polar regions. At the CMB, the maximum normal flux of high southern latitudes is mainly concentrated in one big patch with several less outstanding maxima. Two maxima lie within the TC, one just outside. Weak polar field is observed only at longitudes between 0 and $90^{\circ}$, with an extension of the strong reversed flux patch under the south Atlantic/south African region stretching across the TC boundary.
The evolution of the high latitudes radial field at the CMB back in time, for the northern and the southern hemisphere, is shown by animations provided on our webpage http://www.gfz-potsdam.de/geomagnetic-field/poles. Some (unevenly spaced) snapshots from these animations are shown as representative examples of different field configurations in Fig. 6(b) to (g).

\subsection{Northern hemisphere}

In the northern hemisphere, the CM4 model suggests that the evolution of the prominent Canadian flux lobe mainly corresponds to a slow eastward rotational movement since 1960. This is, however, not confirmed as a longer-term movement by gufm1. In contrast, similar to the behaviour also seen for the second, Siberian flux lobe in CM4, gufm1 suggests that the prominent flux lobes remain more or less stationary on centennial time scales (Bloxham and Jackson, 1992), with modest changes to both east and west and occasional absorption or release of smaller flux maxima. The region within the TC remains a region of weak flux, including reversed patches. The CM4 model predicts one rather large reversed patch stretching across the rotation axis, which splits into two parts in 1975. The part just north of Scandinavia shrinks in the following years and disappears by 1996 . However, the re-appearance of an almost identical patch at 2000 in the CHAOS, but not the CM4 model casts some doubt on the full reliability of such small-scale details. Going further back in time, a reversed flux patch within the TC appears for the first time in gufm 1 at about 1730 . As it evolves it only roughly resembles the one observed in CM4 for the overlapping time span. No reversed flux patches in the polar region appear in gufm 1 prior to 1730 (Fig. 6(b)).

According to the CALS7K.2 model the prominent structure of strong normal flux lobes outside the TC and significantly weaker flux within the northern hemisphere is not persistent over longer time scales. Large areas of strong normal flux in each hemisphere are a characteristic of a dipole dominated field, but the distribution of flux maxima might have changed significantly. In fact the CALS7K.2 model suggests that the current pattern of two distinct northern flux lobes only evolved at about 1600 (i.e. the earliest epochs of the gufm 1 model), while for $2500 \mathrm{yr}$ before (i.e. since $900 \mathrm{BC}$ ) the magnetic flux was similarly strong near the rotation axis (Fig. 6(c)). Between 900 BC and $1800 \mathrm{BC}$ a pair of two distinct flux lobes existed, but at longitudes about $90^{\circ}$ off from the locations of the current ones (Fig. 6(d)). For $1000 \mathrm{yr}$ before, the maximum flux was concentrated in one area mainly outside the TC, which moved around in the European-Asian longitudinal sector (Fig. 6(e)). From 2800 BC back to about 3500 BC, when the flux was generally weaker during the time of weaker dipole moment, the model shows again maximum flux in one larger region including the TC (Fig. 6(f)). Between about $4400 \mathrm{BC}$ and $3500 \mathrm{BC}$, however, flux within the TC was weak again, with 2 to 4 maxima around, approximately at the positions of the current flux lobes and of the ones observed between 900 BC and 1800 BC (Fig. 6(g)).

Reversed flux patches near the polar regions are observed very rarely in the CALS7K.2 model. The earliest one appears around $2260 \mathrm{BC}$ for a few decades only, in fact at the limit of effective temporal resolution of the model. This is 
(a) $\mathrm{B}_{\mathrm{r}}$
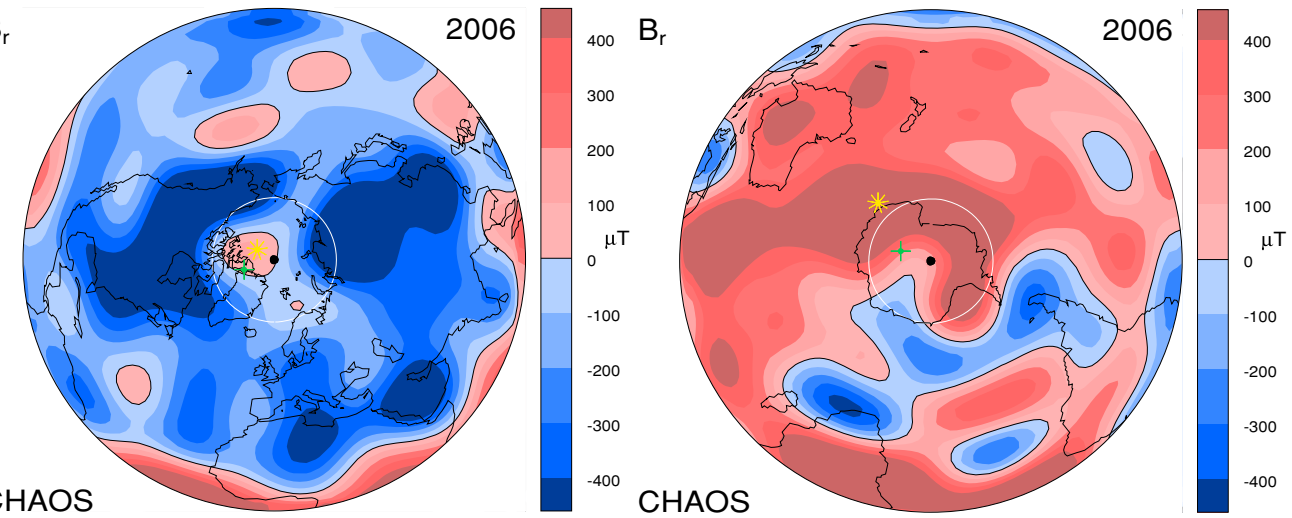

(b) $\mathrm{B}_{\mathrm{r}}$
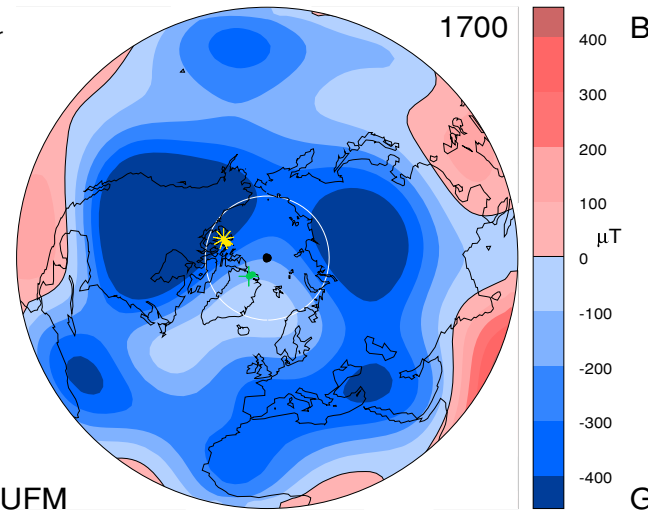

$B_{r}$

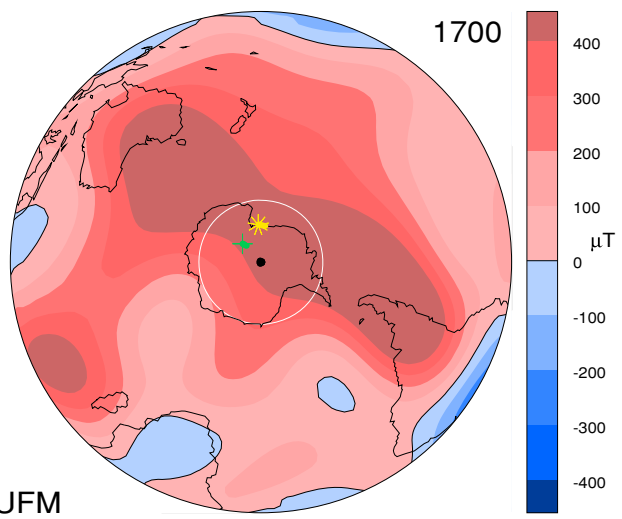

(c) $B_{r}$
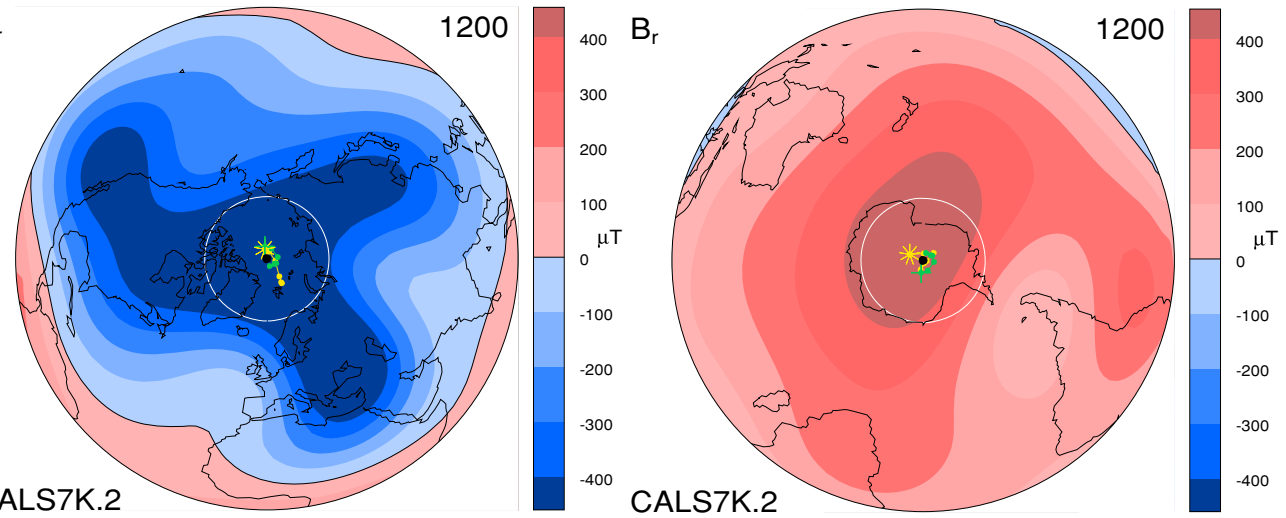

(d) $B_{r}$
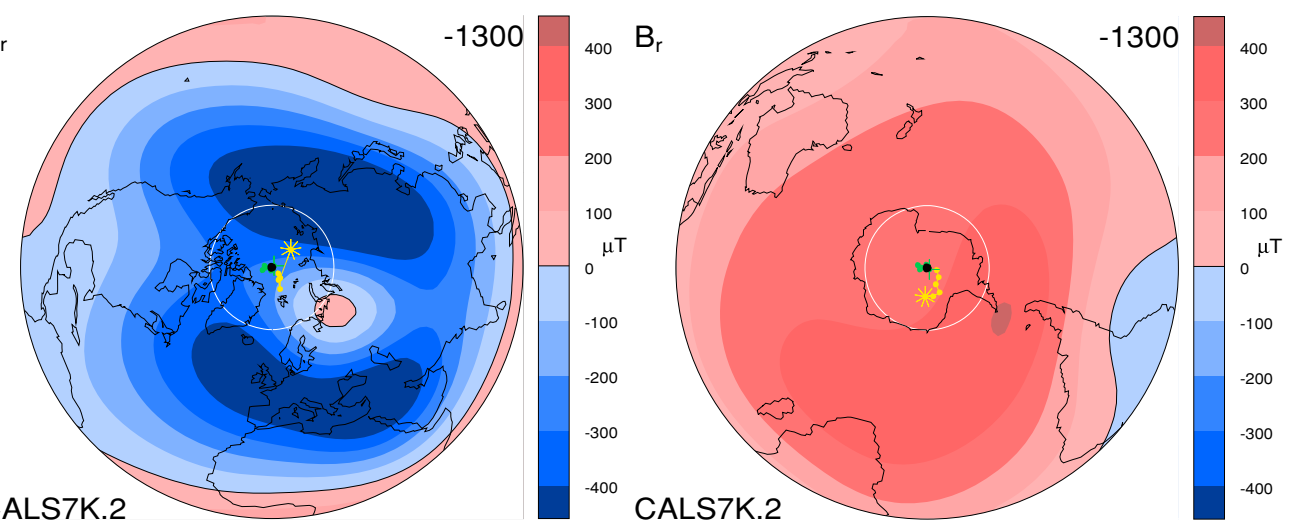

Fig. 6. Snapshots of radial magnetic field $\left(B_{r}\right)$ at the CMB, with locations of magnetic (yellow star) and geomagnetic (green cross) poles at the Earth's surface overlaid (left northern, right southern hemisphere). The pole marks have tails from their previous positions during the preceding 50 and $500 \mathrm{yr}$ in panels from the gufm1 and CALS7K.2 model, respectively. The rotation axis, i.e. geographic pole is marked by a black dot, the inner core TC at the CMB or projected to the Earth's surface by a white circle. (a) CHAOS predictions for 2006, (b) gufm1 predictions for 1700, (c) CALS7K.2 predictions for AD 1200, (d) CALS7K.2 predictions for 1300 BC. (e) CALS7K.2 predictions for $2500 \mathrm{BC}$, (f) CALS7K.2 predictions for $3000 \mathrm{BC}$, (g) CALS7K.2 predictions for $4200 \mathrm{BC}$. 
(e) $B_{r}$

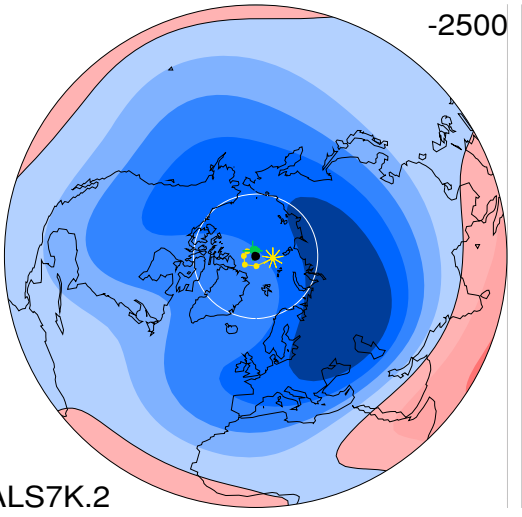

(f) $\mathrm{B}_{\mathrm{r}}$

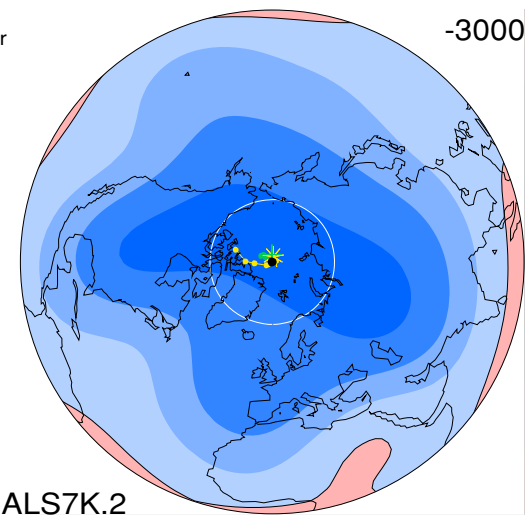

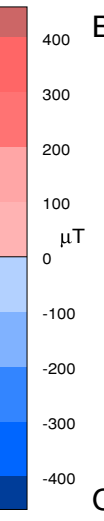

${ }_{400} \mathrm{Br}_{\mathrm{r}}$
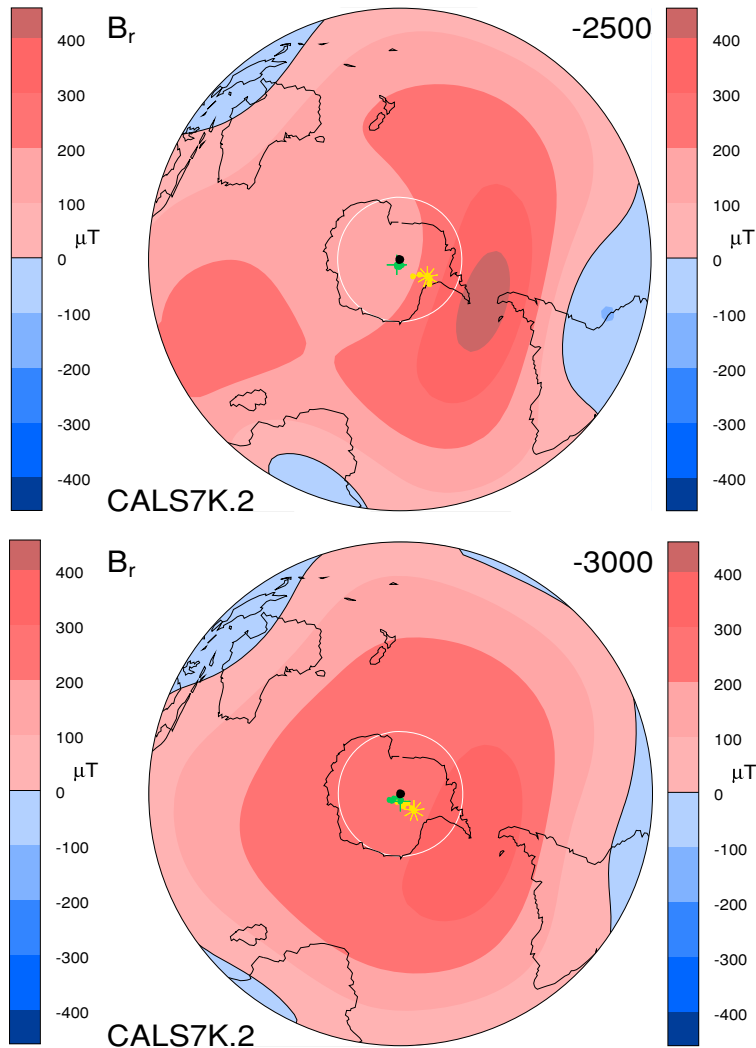

$B_{r}$

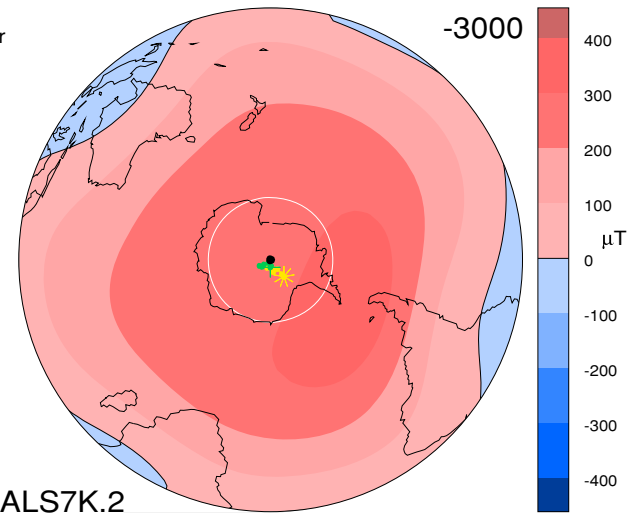

$\mathrm{B}_{\mathrm{r}}$

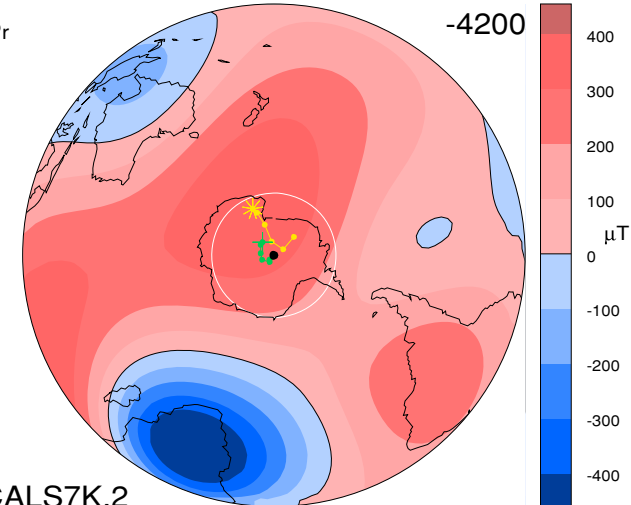

Fig. 6. (continued).

at the time when there is only one strong normal flux maximum moving around the TC, and the small reversed patch occurs in western Greenland, just inside the TC boundary. A larger reversed patch persists from $1340 \mathrm{BC}$ to 1060 BC in northern Russia, mostly outside the TC, although again during a time when the field inside the TC is weak (Fig. 6(d)). Finally, a reversed patch evolves from 1860 to the end of the model in 1950, first appearing near Iceland. This probably is the representation of the patch observed in gufm1 since 1730. In contrast to gufm1, CALS7K shows this patch mostly just outside the TC, but the longitudes agree with the gufm1 patch.

\subsection{Southern hemisphere}

In the southern hemisphere, the general pattern of strong normal flux and a small sector of weak flux, including reversed, with all these features clearly crossing the TC boundary has changed little during the past decades, when the CHAOS, CM4 and gufm1 model are in good to rea- sonable agreement. The observed changes mainly are aggregations or separations of small flux patches and maxima, but exact details, e.g. of the time when two reversed flux patches connect to one, are again not unambiguously resolved when comparing the overlapping models. The gufm1 model predicts that the current pattern started to evolve about 1750, with reversed flux appearing in the nearpolar region early in the 20th century. Before, one large area of normal flux is observed in and around the TC, stronger in the Pacific hemisphere and weaker on the Atlantic/Indian Ocean side (Fig. 6(b)).

The CALS7K. 2 model does not resolve the current pattern with the weak field sector, but shows a large area of normal flux with one varying maximum over the TC and adjacent areas since 1400 B.C. (Fig. 6(c) and (d)). Before, since about $2800 \mathrm{BC}$ the radial field was weaker inside the TC than around, with stronger field for some times fully, for some partly encircling the weak area (Fig. 6(e)). In the 
earlier times, back to $4500 \mathrm{BC}$ the maximum of one large normal flux area for most of the time again included at least parts of the region inside the TC (Fig. 6(f) and (g)). It is striking that when the maximum is strongest, it tends to lie at South American longitudes for the whole $7 \mathrm{kyr}$, according to the CALS7K.2 model. No reversed flux patches appear in the south polar region in this model.

\section{Discussion}

\subsection{Geomagnetic poles}

The behaviour of the geomagnetic poles over the past 2 and $10 \mathrm{kyr}$ has been studied twice previously (Merrill and McElhinny, 1983; Ohno and Hamano, 1992). The results from these studies agree well, but our results for $7 \mathrm{kyr}$ are quite different for much of the time. This is not surprising because the geomagnetic poles of the previous studies in fact are averaged virtual geomagnetic poles (VGPs). VGPs are obtained from palaeodirectional data under the assumption that the field was simply a tilted dipole. Any non-dipole field contributions are mapped into dipole field and bias the location of a VGP with respect to the true geomagnetic pole. To minimise this problem, VGP results from different locations are averaged, but available data are hardly well-distributed over the whole Earth. Ohno and Hamano (1992) already state that their results mainly reflect the geomagnetic variation in northern hemisphere middle to high latitudes, where most of the data come from. Our analysis confirms a westward movement of the dipole axis for the recent $400 \mathrm{yr}$ and a strong eastward swing before, but prior to AD 800 the CALS7K.2 model suggests that there is no predominant westward trend as suggested by the VGPs, but rather more variability in longitudinal movement. Large and strongly varying dipole tilts reported by Ohno and Hamano (1992) prior to 1600 back to $1800 \mathrm{BC}$ are not confirmed by the CALS7K.2 model. On the contrary, this model suggests that the dipole axis was tilted less than today for most of the $7 \mathrm{kyrs}$, and stayed well within the TC.

Figure 4 might suggest that the dipole tends to tilt more strongly during times of weak dipole moment. Indeed, this was generally weak from 5000 to $2000 \mathrm{BC}$, but strong from $1000 \mathrm{BC}$ to AD 1000 and currently is decreasing. However, this impression does not hold up to a rigorous correlation analysis. Particularly the dipole tilt is currently larger than ever before during the studied time interval, while the dipole moment is still stronger than on the $7 \mathrm{kyr}$ average.

\subsection{Magnetic poles}

The magnetic poles have always raised public interest, although their importance with respect to the geodynamo is less clear. The location of NMP and SMP depends on their distance from the source. At the CMB, the influence of non-dipole field is much stronger than at the Earth's surface, and two northern areas where inclination is nearly $90^{\circ}$ at the $\mathrm{CMB}$ can be located in current high-resolution field models. Far out in the magnetosphere, the non-dipole structure has decayed so much that magnetic and geomagnetic poles practically coincide. The area, where the magnetic field shielding effect against incoming cosmic particles is weakest therefore is determined by a combination of geomagnetic and magnetic poles. Figure 7 shows the magnetic

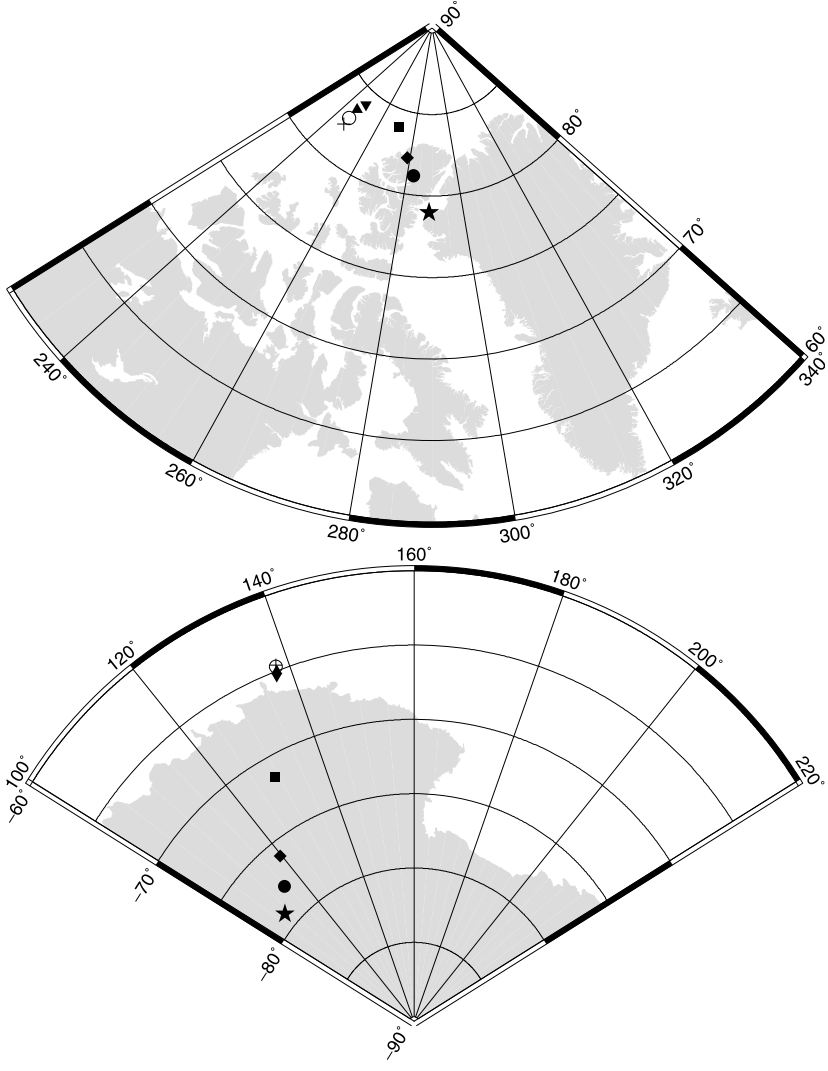

Fig. 7. The magnetic poles at different altitudes: at the Earth's surface (cross), at $100 \mathrm{~km}$ (circle), $300 \mathrm{~km}$ (triangle), $600 \mathrm{~km}$ (inverted triangle), and at one (square), 3 (diamond) and 6 (full circle) Earth radii above. The star marks the geomagnetic pole or dipole axis position.

pole locations at different altitudes above the Earth. In the near atmosphere (less than $100 \mathrm{~km}$ altitude) and the ionosphere (up to about $400 \mathrm{~km}$ altitude) the magnetic pole positions are still similar to the ones at the surface, while they change significantly over the distances of a few Earth radii within the magnetosphere. The variation of location with altitude close to the surface is stronger for the NMP than the SMP, which is a consequence of the smaller-scale field structure currently observed in the northern polar region. At intermediate distances of about 1 Earth radius the change of pole location with altitude is stronger in the southern hemisphere.

On time scales longer than the most recent 4 centuries we have to be careful with conclusions about the magnetic poles. The CALS7K.2 model does not seem to represent the magnetic pole positions reasonably. We performed a simple exercise to obtain a better understanding of the model limitation, and investigated what an effect truncating the gufm 1 model at low spherical harmonic degrees has on the magnetic pole positions. Figure 8 shows the results for truncation at degree 4, 3 and even 2 in comparison to the original gufm 1 predictions and the CALS7K.2 predictions. The result is somewhat surprising: directions and changes of pole motion are a feature of the large-scale field, already contained mainly in quadrupole (for the SMP) or octupole (for the NMP) contributions. The higher degrees determine basically only the exact latitudes and longitudes.

At a first glance, the CALS7K.2 predictions in this figure 

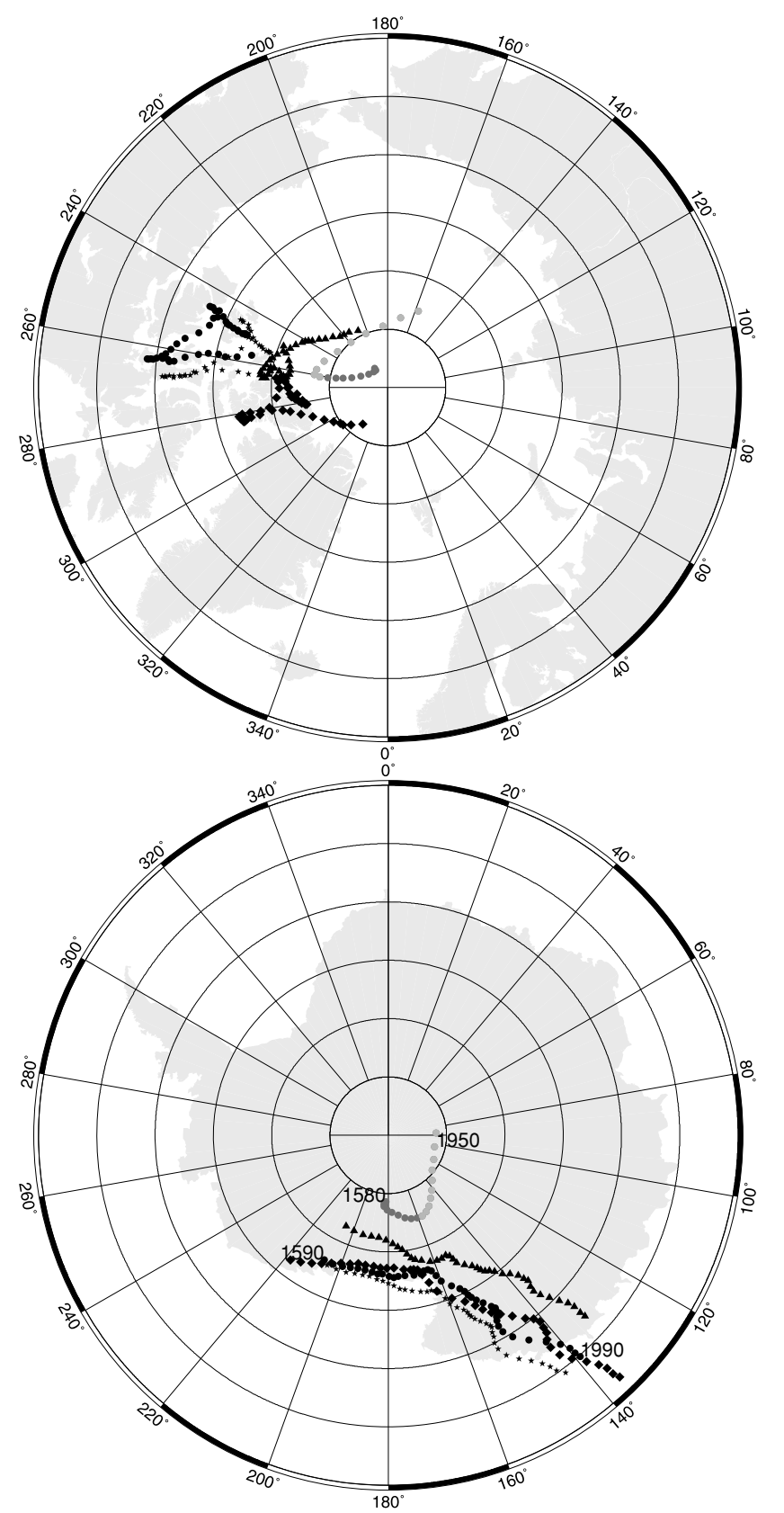

Fig. 8. Magnetic pole location 1590 to 1990 from gufm1 (dots) and from the same model truncated at spherical harmonic degree 4 (stars), 3 (diamonds) and 2 (triangles). Gray are the CALS7K.2 predictions from 1580 to 1950 , with the time interval since 1750 , which might be significantly influenced by spline edge effects in light gray.

look significantly different. However, a closer comparison reveals that yet there is some agreement, considering again that edge effects due to the spline basis and sparse data towards the ends of the model exist, for the recent end from approximately 1750 onwards. Similar edge effects might influence gufm1 for the earliest few decades. For the remaining overlapping period (only the dark gray CALS7K.2 predictions in Fig. 8) the general trends of movement in both longitude and latitude mainly agree with the gufm1 predictions. It is only surprising that the CALS7K.2 predictions are much more offset in latitude than even those containing no more than dipole and quadrupole contributions from gufm1. It is difficult to make inferences about the reliability of the CALS7K.2 predictions of pole velocities based on this comparison because of the low temporal resolution of the millennial scale model. Indeed, not much variation is observed between the early 17 th and mid-18th century. However, we assume that relative differences in velocity predicted by CALS7K.2 result from reliable changes in pole behaviour, i.e. the NMP moved faster than on average at $4500 \mathrm{BC}$ and $1300 \mathrm{BC}$ (Fig. 5). Note, however, that accelerations lasting less than a century, as the current one probably will do, are generally not resolved in the $7 \mathrm{kyr}$ model.

The magnetic poles show significantly stronger deviations from the rotation axis than the geomagnetic poles on all time-scales. Nevertheless, their trace at the Earth's surface generally stayed within the the TC boundary projected to the surface, i.e. the approximate surface region influenced by the core field inside the TC. Note that this is not a sharp boundary at the Earth's surface and that the magnetic pole location depends on distance from the CMB. With the uncertainties on exact pole locations predicted by CALS7K.2, however, it is likely that occasional movements up to or crossing the TC boundary have occured, e.g. at $4100 \mathrm{BC}$ in the southern or $350 \mathrm{BC}$ in the northern hemisphere (Fig. 4), similar to 1920 and 1860 AD in Antarctica and Canada, respectively.

\subsection{Polar fields}

The current and past field behaviour described in Section 4 shows no persistent differences between the areas inside and outside the TC. Significant changes of flux bundles over centennial to millennial time scales are in principle agreement with theoretical results by Bloxham (2002), who found that heat flux variations imposed on a numerical dynamo model cause significant changes in flux concentrations. However, the latitude of the heat flux variation was fixed in that study, leaving open the question of influence of the inner core and TC on the location of flux maxima. Our results suggest that significant weaker flux within the TC is not a persistent feature of the field. One might caution that the CALS7K.2 model could be missing weak field structure in polar regions at least at certain times, due to the limited resolution and particularly sparse data from high latitudes. However, the current southern hemisphere polar field with two flux maxima within the TC rather supports the possibility that the influence of inner core and TC boundary on the geodynamo process could be less important than heat flux variations in determination of field morphology, and that the (general large-scale) field features predicted by CALS7K.2 could be reliable. More detailed structure like particularly the presence or absence of rather small reversed flux patches in weak flux regions, however, is very likely not resolved well enough in CALS7K.2 to permit any interpretation.

Our results suggest that an asymmetry not only in field morphology, but also in amount of field changes persisted during the past $7 \mathrm{kyr}$, with both more structure and variation in the northern hemisphere. Unfortunately, the data basis for CALS7K.2 is strongly biased towards the northern hemisphere (Korte and Constable, 2005b), with significant amounts of southern hemisphere data coming from two narrow longitudinal sectors only, namely the Southern American and western Australian/New Zealand regions. 
The lower agreement of southern than northern hemispheric field from CALS7K.2 to gufm 1 confirms the suspicion that the southern polar field is not resolved to the same degree as the northern field in the millennial scale model. Therefore we cannot be completely confident about the inferences on the persistence of asymmetric field behaviour over long times. New southern hemisphere data are needed for a confirmation.

\section{Conclusions}

Although our study of truncated gufm1 model predictions suggests that the general magnetic pole evolution is determined by large scale field, we found no significant correlations between NMP, SMP and dipole axis behaviour. We conclude that the dipole axis changes independently from other field contributions. The different behaviour of NMP and SMP is not surprising, given the asymmetry of northern and southern hemisphere field, and particularly polar field morphology and variation. The independent pole behaviour might be taken as an indication that significant differences exist between the field inside the northern hemisphere TC, inside the southern one and outside. However, we could not find any clear evidence for the TC signature when studying the polar field evolution at the CMB. Weak and reversed flux areas within the TC with strong flux lobes around do not seem to be a feature of the field persistent over more than a few centuries. The CALS7K.2 model suggests strong changes in flux distribution over 7 kyr. Moreover, current high-resolution models show strong normal flux within the southern hemisphere TC.

The abrupt increase in velocity of the NMP at the Earth's surface is linked to a reversed polar flux patch at the CMB, as also noted by Olsen and Mandea (2007). The current position of the NMP is straight above this patch. For several centuries it had been above the northern margin of the strong normal Canadian flux patch. The movement towards higher latitudes must have been triggered by the opposite, Siberian normal flux patch getting stronger. At about 1950 the NMP was above the null-flux curve, getting close to the maximum. This leads to the conclusion that most likely the NMP will slow down after crossing the maximum and will continue to decelerate for a while, if it keeps its current direction of movement towards the location of the Siberian flux lobe. The CHAOS model suggests that the NMP has just crossed the position of maximal reversed flux at the CMB and indeed Olsen and Mandea (2007) find in an extended model that the pole recently started to decelerate. However, if the flux concentration in the two strong normal flux lobes should change significantly and the Canadian one become more dominant once again, the NMP would reverse its direction of motion, probably quite abruptly as it already did several times in the past. The velocity would remain high as long as its position remains above a region of strong reversed flux. The current knowledge about the geodynamo process is insufficient to predict magnetic core field changes and continuous measurements remain indispensable.

Acknowledgments. We thank Susan Macmillan and Tadahiro Hatakeyama for constructive reviews that helped to improve the original manuscript. Maps were created using the free software
GMT (Wessel and Smith, 1998), and Magmap and Color by Robert L. Parker.

\section{References}

Aurnou, J., S. Andreadis, L. Zhu, and P. Olson, Experiments on convection in the Earth's core tangent cylinder, Earth Planet. Sci. Lett., 212, 119 134, 2003.

Bard, E. and G. Delaygue, Comment on "Are there connections between the Earth's magnetic field and climate?" by V. Courtillot, Y. Gallet, J.-L. LeMouël, F. Fluteau, A. Genevey, EPSL, 253, 328, 2007, Earth Planet. Sci. Lett., 265, 302-307, 2007.

Barton, C. E., Position of the south magnetic pole, January 1986, Eos Trans. $A G U, \mathbf{6 8}(12), 162,1987$.

Barton, C. E., More on the south magnetic pole, Eos Trans. AGU, 69(4), 50, 1988.

Barton, C., Survey tracks current position of south magnetic pole, Eos Trans. AGU, 83(27), 291, 2002.

Bloxham, J., Time-independent and time-dependent behaviour of highlatitude flux bundles at the core-mantle boundary, Geophys. Res. Lett., 29, 1854, doi:10.1029/2001GL014543, 2002.

Bloxham, J. and D. Gubbins, The secular variation of Earth's magnetic field, Nature, 317, 777-781, 1985.

Bloxham, J. and A. Jackson, Time-dependent mapping of the magnetic field at the core-mantle boundary, J. Geophys. Res., 97, 19,537-19,563, 1992.

Cardin, P. and P. Olson, Chaotic thermal convection in a rapidly rotating spherical shell: consequences for flow in the outer core, Phys. Earth Planet. Inter., 82, 235-259, 1994.

Carrigan, C. R. and F. H. Busse, An experimental and theoretical investigation of the onset of convection in rotating spherical shells, J. Fluid Mech., 126, 287-305, 1983.

Constable, C. and M. Korte, Is Earth's magnetic field reversing?, Earth Planet. Sci. Lett., 246, 1-6, 2006.

Courtillot, V., Y. Gallet, J.-L. LeMouël, F. Fluteau, and A. Genevey, Are there connections between the Earth's magnetic field and climate, Earth Planet. Sci. Lett., 253, 328-339, 2007.

Dawson, E. and L. R. Newitt, The magnetic poles of the Earth, J. Geomag. Geoelectr., 34(4), 225-240, 1982.

Dormy, E., A. M. Soward, C. A. Jones, D. Jault, and P. Cardin, The onset of thermal convection in rotating spherical shells, J. Fluid Mech., 501, 43-70, 2004.

Gubbins, D., Mechanism for geomagnetic polarity reversals, Nature, 326, 167-169, 1987.

Gubbins, D. and J. Bloxham, Morphology of the geomagnetic field and implications for the geodynamo, Nature, 325, 1521-1524, 1987.

Gubbins, D., A. L. Jones, and C. C. Finlay, Fall in Earth's magnetic field is erratic, Science, 312, 900-902, 2006.

Hulot, G., C. Eymin, B. Langlais, M. Mandea, and N. Olsen, Small-scale structure of the geodynamo inferred from Oersted and Magsat satellite data, Nature, 416, 620-623, 2002.

Jackson, A., A. R. T. Jonkers, and M. R. Walker, Four centuries of geomagnetic secular variation from historical records, Phil. Trans. R. Soc. Lond. A, 358, 957-990, 2000.

Korte, M. and C. G. Constable, Continuous geomagnetic field models for the past 7 millennia: 2. CALS7K, Geochem. Geophys. Geosyst., 6, Q02H16, doi:10.1029/2004GC000801, 2005a.

Korte, M. and C. G. Constable, Continuous geomagnetic field models for the past 7 millennia: A new global data compilation, Geochem. Geophys. Geosyst., 6, Q02H15, doi:10.102/2004GC000800, 2005b.

Korte, M. and C. G. Constable, Spatial and temporal resolution of millennial scale field models, Adv. Space Res., 41, 57-69, 2008.

Langel, R. A., The main field, in Geomagnetism, 1, edited by J. A. Jacobs, 249-512, Academic Press, Orlando, 1987.

Mandea, M. and E. Dormy, Asymmetric behaviour of magnetic dip poles, Earth Planets Space, 55, 153-157, 2003.

Merrill, R. T. and M. W. McElhinny, The Earth's magnetic field: Its history, origin and planetary perspective, 401 pp, Academic Press, London, 1983.

Newitt, L. R. and C. E. Barton, The position of the north magnetic dip pole in 1994, J. Geomag. Geoelectr., 48, 221-232, 1996.

Newitt, L. R., M. Mandea, L. A. McKee, and J.-J. Orgeval, Recent acceleration of the north magnetic pole linked to magnetic jerks, Eos Trans. $A G U, \mathbf{8 3}(35), 381-389,2002$.

Ohno, M. and Y. Hamano, Geomagnetic poles over the past 10,000 years, Geophys. Res. Lett., 19, 1715-1718, 1992. 
Olsen, N. and M. Mandea, Will the magnetic north pole move to siberia?, Eos Trans. AGU, 88(29), 293, 2007.

Olsen, N., H. Lühr, T. J. Sabaka, M. Mandea, M. Rother, L. ToffnerClausen, and S. Choi, CHAOS-A model of the Earth's magnetic field derived from CHAMP, orsted, and SAC-C magnetic satellite data, Geophys. J. Int., 166(1), 67-75, 2006.

Olson, P., The disappearing dipole, Nature, 416, 591-594, 2002.

Olson, P. and J. Aurnou, A polar vortex in the Earth's core, Nature, 402, 170-173, 1999.

Pais, A. and G. Hulot, Length of day decade variations, torsional oscillations and inner core superrotation: evidence from recovered core surface zonal flows, Phys. Earth Planet. Inter., 118, 291-316, 2000.
Riedel, K. S. and A. Sidorenko, Minimum bias multiple taper spectral estimation, IEEE Trans. Signal Process., 43, 188-195, 1995.

Sabaka, T. J., N. Olsen, and M. E. Purucker, Extending comprehensive models of the Earth's magnetic field with Ørsted and CHAMP data, Geophys. J. Int., 159, 521-547, 2004.

Wessel, P. and W. H. F. Smith, New, improved version of the generic mapping tools released, Eos Trans. AGU, 79, 579, 1998.

M. Korte (e-mail: monika@gfz-potsdam.de) and M. Mandea 